\title{
Release of Civil Registry Causes of Death Data in Iran (2015 to 2019) - Expectations and Doubts!
}

\author{
Hooman Tadbiri, MD, MSc, MPH${ }^{1}$; Maziar Moradi-Lakeh, MD, MPH ${ }^{2 *}$; Mohsen Naghavi, MD, PhD \\ ${ }^{1}$ Department of Obstetrics, Gynecology and Reproductive Sciences, University of Maryland School of Medicine, Baltimore, MD, USA \\ ${ }^{2}$ Preventive Medicine and Public Health Research Center, Psychosocial Health Research Institute, Iran University of Medical Sciences, \\ Tehran, Iran \\ ${ }^{3}$ Institute for Health Metrics and Evaluation, University of Washington, Seattle, WA, USA
}

\begin{abstract}
Background: The National Organization for Civil Registration (NOCR) of Iran has reported causes of death data by ICD-10 chapters for the first time in 2020.

Methods: We used this report to review the share of ICD chapters among all deaths in each province and compare them with the Global Burden of Disease (GBD) study.

Results: There are major changes in the distribution of causes of death between 2017 and 2018, especially in D50-D89 (Diseases of the blood and blood-forming organs and certain disorders involving the immune mechanism) from $0.76 \%$ to $38.94 \%$, and 100-199 (Diseases of the circulatory system) from 39.27\% to $7.09 \%$. Such dramatic changes are probably the results of changes in coding practices or definitions or issues in analysis.

Conclusion: Causes of death reports should be timely, clear, and robust on methods. They should contain a minimum level of details, at least 3-digit ICD codes to be useful for public health and medical professionals.

Keywords: Cause of Death, Iran, Vital Statistics

Cite this article as: Tadbiri H, Moradi-Lakeh M, Naghavi M. Release of civil registry causes of death data in iran (2015 to 2019) expectations and doubts!. Arch Iran Med. 2021;24(10):741-746. doi: 10.34172/aim.2021.109
\end{abstract}

Received: April 13, 2021, Accepted: May 5, 2021, ePublished: October 1, 2021

\section{Introduction}

Civil registration and vital statistics (CRVS) systems register vital events, including births, deaths, marriages, and divorces. CRVS systems use this data to compile vital statistics, such as mortality statistics and causes of death. ${ }^{1}$ Mortality statistics are widely used by policymakers, government officials, researchers, and the private sector. ${ }^{2}$ Uses of this information include but are not limited to measuring health indicators and public health goals, enacting and evaluating policies and regulations, and allocating resources to health and research. ${ }^{3}$ However, CRVS systems in many countries have major issues such as under-coverage of vital events or misclassification of causes of deaths and need substantial improvement. ${ }^{4}$

The United Nations Economic and Social Commission for Asia and the Pacific (ESCAP) convened the first Ministerial Conference on CRVS in Asia and the Pacific in 2014. This conference led to endorsement of a framework containing a set of goals to improve the CRVS systems in the region by 2024 and declaration of the years 2015 to 2024 to be the CRVS Decade for Asia and the Pacific. The $3^{\text {rd }}$ goal to be achieved in this decade was "accurate, complete and timely vital statistics (including on causes of death), based on registration records, are produced and disseminated". Iran, like the other collaborating countries, is committed to achieving several national targets under the $3^{\text {rd }}$ goal of this framework, which include but are not limited to ${ }^{6}$ :

- Producing annual nationally representative statistics on deaths - disaggregated by age, sex, cause of death defined by the International Classification of Diseases $(\mathrm{ICD})^{7}$ (latest version as appropriate), geographic area and administrative subdivision - from registration records or other valid administrative data sources by 2020.

- Having an underlying cause of death code derived from the medical certificate according to the ICD standards for at least 85 percent of deaths occurring in health facilities or with the attention of a medical practitioner by 2024 (and at least 50\% of deaths taking place outside of a health facility and without the attention of a medical practitioner).

- Reducing the proportion of deaths coded to ill-defined codes to less than 10 percent by 2024 compared with the baseline year (2015).

- Making key summary tabulations of vital statistics on births and deaths available in the public domain in electronic format annually by 2015 , and within one calendar year.

- Making key summary tabulations of vital statistics on causes of death available in the public domain in electronic format annually by 2020 , and within two 
calendar years.

In Iran, the National Organization for Civil Registration (NOCR) was established in 1918 and is responsible for CRVS in the country. ${ }^{8}$ The NOCR has included causes of death data in their annual vital statistics report since 2006. ${ }^{9}$ There is a parallel system at the Ministry of Health and Medical Education (MOHME) in Iran that assigns causes of death based on a combination of clinical records, verbal autopsies, and data from cemeteries. Although the NOCR data has better coverage on mortality envelopes, causes of deaths in the MOHME system are more accurate. $^{10,11}$ However, MOHME reports on causes of deaths have a considerable delay and are not available on public domains. In dramatic progress toward the ESCAP goals for the CRVS Decade, the NOCR in 2019 reported causes of death data for 2018 in public domain by ICD chapters for the first time. ${ }^{12}$ In 2020, the NOCR published the causes of death data from 2015 to 2019 grouped by ICD-10 chapters. ${ }^{13}$

In this study, we reviewed the recent causes of death data from NOCR and compared it with another source to assess its accuracy.

\section{Materials and Methods}

The "Vital Statistics Yearly Summary 2015-19" report from NOCR was published online in $2020 .{ }^{13}$ We extracted the number of deaths under each ICD-10 chapter from this report for each of the provinces for each of the five consecutive years. We used this data to calculate the share of each cause of death from the total number of deaths registered in each province from 2015 to 2019. We also aggregated this data and performed the calculations at the national level.

We compared the causes of death data released by NOCR with the causes of death estimates from the Global Burden of Disease (GBD) study. The GBD study is a worldwide epidemiological study, led by the Institute for Health Metrics and Evaluation (IHME). The GBD study provides global health data, including the causes of death in more than 200 countries, and their latest results (GBD 2019) was published in 2020..$^{14,15}$ We used the GBD Compare Data Visualization tool ${ }^{16}$ to compare the top causes of death in Iran between 2015 and 2019. Then, we identified the corresponding ICD-10 chapter for each of the causes of death reported by the GBD study for 2019 and compared it with the NOCR report for 2019. The GBD study reports causes of death at four different levels. The first level has the three categories of "communicable, maternal, neonatal, and nutritional diseases", "noncommunicable diseases", and "injuries" which are not translatable into ICD-10 chapters. These three categories in level 1 are divided into 21 categories in level 2, into 79 categories in level 3, and into 101 categories in level 4. Most of the level 2 categories from the GBD study fall under an ICD-10 chapter. We used the level 3 estimates from the GBD study and split the corresponding level 2 category if it fell under more than one ICD-10 chapter.

\section{Results}

Figure 1 and Table 1 show the share of each cause of death from the total deaths registered at the national level in Iran in each of these five years. This information is also available at the provincial level in Supplementary file. As is clear in Figure 1, there is a major change in the distribution of causes of death between 2017 and 2018 which is also recognizable in all provinces (Supplementary file 1). Figure 2 shows how the ranking of causes of deaths registered in Iran has changed from 2015 to 2019.

The most significant absolute change is related to the codes D50-D89 (representing "diseases of the blood and blood-forming organs and certain disorders involving the immune mechanism") which increased from less than $1 \%$ in 2015-2017 to nearly $40 \%$ in $2018-2019$. The second most significant change is related to the codes I00-I99 (representing "diseases of the circulatory system") which decreased from nearly $40 \%$ in $2015-2017$ to around 7\% in 2018-2019. Other notable changes are the codes R00-R99 (representing "symptoms, signs, and abnormal clinical and laboratory findings, not elsewhere classified") that decreased from around $11 \%$ in $2015-2017$ to less than $1 \%$

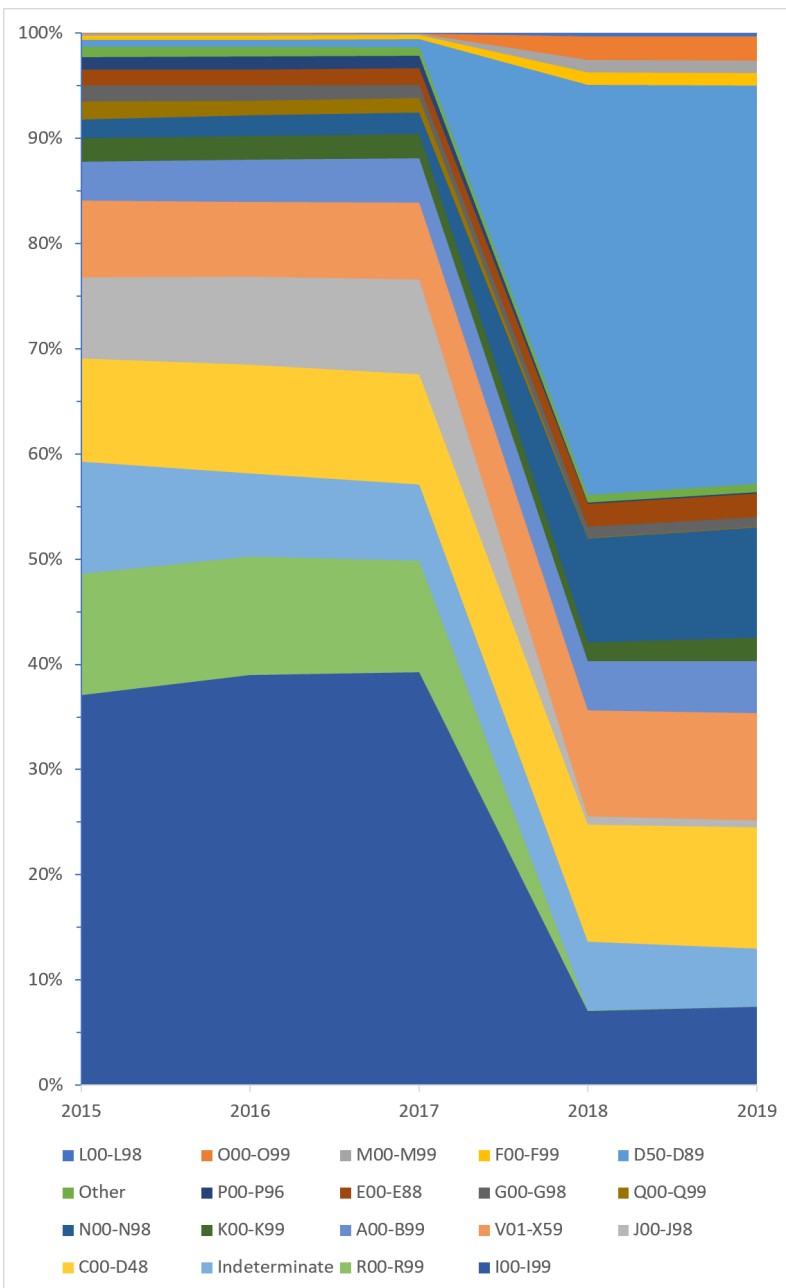

Figure 1. Distribution of Causes of Deaths at National Level in Iran Based on the International Classification of Disease (ICD 10) Chapters, National Organization for Civil Registration (2015-2019). 
Table 1. Causes of Death at National Level in Iran (2015-2019) Based on the International Classification of Diseases (ICD) Chapters: Reports of the National Organization for Civil Registration (NOCR)

\begin{tabular}{|c|c|c|c|c|c|c|}
\hline ICD-10 Codes & Title & 2015 & 2016 & 2017 & 2018 & 2019 \\
\hline A00-B99 & Certain infectious and parasitic diseases & $3.66 \%$ & $4.01 \%$ & $4.23 \%$ & $4.67 \%$ & $4.96 \%$ \\
\hline C00-D48 & Neoplasms & $9.84 \%$ & $10.38 \%$ & $10.47 \%$ & $11.12 \%$ & $11.54 \%$ \\
\hline D50-D89 & $\begin{array}{l}\text { Diseases of the blood and blood-forming organs and } \\
\text { certain disorders involving the immune mechanism }\end{array}$ & $0.67 \%$ & $0.67 \%$ & $0.76 \%$ & $38.94 \%$ & $37.85 \%$ \\
\hline E00-E88 & Endocrine, nutritional, and metabolic diseases & $1.50 \%$ & $1.49 \%$ & $1.60 \%$ & $2.17 \%$ & $2.27 \%$ \\
\hline F00-F99 & Mental and behavioral disorders & $0.36 \%$ & $0.39 \%$ & $0.37 \%$ & $1.22 \%$ & $1.14 \%$ \\
\hline G00-G98 & Diseases of the nervous system & $1.55 \%$ & $1.47 \%$ & $1.26 \%$ & $1.05 \%$ & $0.92 \%$ \\
\hline 100-199 & Diseases of the circulatory system & $37.07 \%$ & $38.95 \%$ & $39.27 \%$ & $7.09 \%$ & $7.45 \%$ \\
\hline J00-J98 & Diseases of the respiratory system & $7.68 \%$ & $8.35 \%$ & $8.99 \%$ & $0.80 \%$ & $0.66 \%$ \\
\hline K00-K99 & Diseases of the digestive system & $2.25 \%$ & $2.27 \%$ & $2.31 \%$ & $1.79 \%$ & $2.18 \%$ \\
\hline LO0-L98 & Diseases of the skin and subcutaneous tissue & $0.03 \%$ & $0.03 \%$ & $0.04 \%$ & $0.31 \%$ & $0.32 \%$ \\
\hline M00-M99 & $\begin{array}{l}\text { Diseases of the musculoskeletal system and connective } \\
\text { tissue }\end{array}$ & $0.22 \%$ & $0.20 \%$ & $0.15 \%$ & $1.19 \%$ & $1.23 \%$ \\
\hline No0-N98 & Diseases of the genitourinary system & $1.76 \%$ & $1.94 \%$ & $2.05 \%$ & $9.89 \%$ & $10.53 \%$ \\
\hline O00-O99 & Pregnancy, childbirth, and the puerperium & $0.05 \%$ & $0.05 \%$ & $0.04 \%$ & $2.25 \%$ & $2.30 \%$ \\
\hline P00-P96 & Certain conditions originating in the perinatal period & $1.20 \%$ & $1.28 \%$ & $1.17 \%$ & $0.13 \%$ & $0.15 \%$ \\
\hline Q00-Q99 & $\begin{array}{l}\text { Congenital malformations, deformations, and } \\
\text { chromosomal abnormalities }\end{array}$ & $1.73 \%$ & $1.40 \%$ & $1.36 \%$ & $0.03 \%$ & $0.02 \%$ \\
\hline R00-R99 & $\begin{array}{l}\text { Symptoms, signs, and abnormal clinical and laboratory } \\
\text { findings, not elsewhere classified }\end{array}$ & $11.53 \%$ & $11.28 \%$ & $10.58 \%$ & $0.03 \%$ & $0.03 \%$ \\
\hline V01-X59 & External causes of morbidity and mortality & $7.32 \%$ & $7.08 \%$ & $7.30 \%$ & $10.07 \%$ & $10.19 \%$ \\
\hline Other & & $0.96 \%$ & $0.87 \%$ & $0.82 \%$ & $0.75 \%$ & $0.77 \%$ \\
\hline Indeterminate & & $10.63 \%$ & $7.89 \%$ & $7.24 \%$ & $6.49 \%$ & $5.50 \%$ \\
\hline
\end{tabular}

\section{5 rank}

2019 rank

\begin{tabular}{|c|c|}
\hline 1. I00-I99 (37.07\%) & 1. D50-D89 (37.85\%) \\
\hline 2. R00-R99 (11.53\%) & 2. C00-D48 (11.54\%) \\
\hline 3. Indeterminate $(10.63 \%)$ & 3. N00-N98 (10.53\%) \\
\hline 4. C00-D48 (9.84\%) & 4. V01-X59 (10.19\%) \\
\hline 5. J00-J98 (7.68\%) & 5. I00-I99 (7.45\%) \\
\hline 6. V01-X59 (7.32\%) & 6. Indeterminate $(5.50 \%)$ \\
\hline 7. A00-B99 (3.66\%) & 7. A00-B99 (4.96\%) \\
\hline 8. K00-K99 (2.25\%) & 8. 000-099 (2.30\%) \\
\hline 9. N00-N98 (1.76\%) & 9. E00-E88 (2.27\%) \\
\hline 10. Q00-Q99 (1.73\%) & 10. K00-K99 (2.18\%) \\
\hline 11. G00-G98 (1.55\%) & 11. M00-M99 (1.23\%) \\
\hline 12. E00-E88 (1.50\%) & 12. F00-F99 (1.14\%) \\
\hline 13. P00-P96 (1.20\%) & 13. G00-G98 (0.92\%) \\
\hline 14. Other $(0.96 \%)$ & 14. Other $(0.77 \%)$ \\
\hline 15. D50-D89 (0.67\%) & 15. J00-J98 (0.66\%) \\
\hline 16. F00-F99 (0.36\%) & 16. L00-L98 (0.32\%) \\
\hline 17. M00-M99 (0.22\%) & 17. P00-P96 (0.15\%) \\
\hline 18. 000-099 (0.05\%) & 18. R00-R99 (0.03\%) \\
\hline 19. L00-L98 (0.03\%) & 19. Q00-Q99 (0.02\%) \\
\hline
\end{tabular}

Figure 2. Causes of Death (Percent of Total Deaths) from the National Organization for Civil Registration (NOCR) Report, 2015 vs. 2019. 
in 2018-2019, N00-N99 ("diseases of the genitourinary system") that increased from around 2\% in 2015-2017 to around 10\% in 2018-2019, and J00-J98 ("diseases of the respiratory system") that decreased from around $8 \%$ in 2015-2017 to less than $1 \%$ in 2018-2019.

To compare NOCR data with another estimate, we used GBD 2019 estimates for Iran. Figure 3 shows how the ranking of causes of deaths in Iran has changed from 2015 to 2019 according to the GBD study. Table 2 compares the share of each cause of death in Iran in 2019 from the GBD study estimates with the NOCR report.

The NOCR report attributes $37.85 \%$ of total deaths in Iran in 2019 to ICD codes D50-D89. However, based on GBD study, the most common cause of the deaths under the ICD codes D50-D89 is "hemoglobinopathies" which was the cause for only $0.01 \%$ of total deaths in Iran in 2019. This is by far the largest incompatibility between these two sources. The other large difference between these two sources is the "cardiovascular diseases" that accounts for $44.39 \%$ of total deaths in Iran in 2019 according to the GBD study while the ICD codes I00-I99 account for only $7.45 \%$ of total deaths in the NOCR report.

\section{Discussion}

It seems clear that the coding pattern of registered deaths has changed significantly since 2018 . Real changes with this scale in causes of deaths during five years are not plausible. Those dramatic changes have probably resulted from a change in coding practices or definitions or an issue in analysis.

The NOCR data are pooled from death certificates. Death certificates are legal documents issued upon death, and they have a section dedicated to the cause of death. The chain of diseases or conditions leading to death are listed under this section. The "immediate" cause of death is placed on the first line, followed by the intermediate causes of death in the next lines, and finally, the "underlying" cause of death is placed on the last line. The underlying cause of death (that starts the process which ends in death) is used to code the cause of death. ${ }^{17}$ The certifier in Iran is a physician or forensic medicine authority. ${ }^{6}$ If the cause of death is not coded into ICD codes by the certifier, the NOCR will convert the written phrase to ICD code. ${ }^{18}$ The chapters of ICD-10 are subdivided into 3 -digit codes, and most of those 3-digit codes are further subdivided into 4-digit codes. ${ }^{7}$ Reporting the cause of death aggregated into ICD-10 chapters instead of at least 3 -digit codes is not helpful for public health purposes and could be misleading.

Having the "diseases of the blood and bloodforming organs" as the leading cause at national level is unimaginable. There is no country in the world with this as their top leading cause of death. ${ }^{15,19}$ The increased share of ICD codes D50-D89 to 38\% in NOCR report is clearly an error. Overrepresentation of cardiovascular diseases as
2015 rank

\begin{tabular}{|c|}
\hline 1. Cardiovascular diseases $(42.15 \%)$ \\
\hline 2. Neoplasms (15.86\%) \\
\hline 3. Diabetes \& CKD (6.5\%) \\
\hline 4. Transport injuries (6.25\%) \\
\hline 5. Neurological disorders (4.27\%) \\
\hline 6. Chronic respiratory (4.21\%) \\
\hline 7. Maternal \& neonatal (4.1\%) \\
\hline 8. Unintentional inj (3.32\%) \\
\hline 9. Other non-communicable (2.99\%) \\
\hline 10. Respiratory infections \& TB (2.99\%) \\
\hline 11. Digestive diseases $(2.83 \%)$ \\
\hline 12. Self-harm \& violence $(2.16 \%)$ \\
\hline 13. Substance use $(0.84 \%)$ \\
\hline 14. Other infectious (0.57\%) \\
\hline 15. Enteric infections $(0.38 \%)$ \\
\hline 16. HIV/AIDS \& STIs (0.27\%) \\
\hline 17. Musculoskeletal disorders $(0.15 \%)$ \\
\hline 18. Nutritional deficiencies (0.067\%) \\
\hline 19. Skin diseases $(0.066 \%)$ \\
\hline 20. NTDs \& malaria $(0.027 \%)$ \\
\hline 21. Mental disorders $(0.000097 \%)$ \\
\hline
\end{tabular}

2019 rank

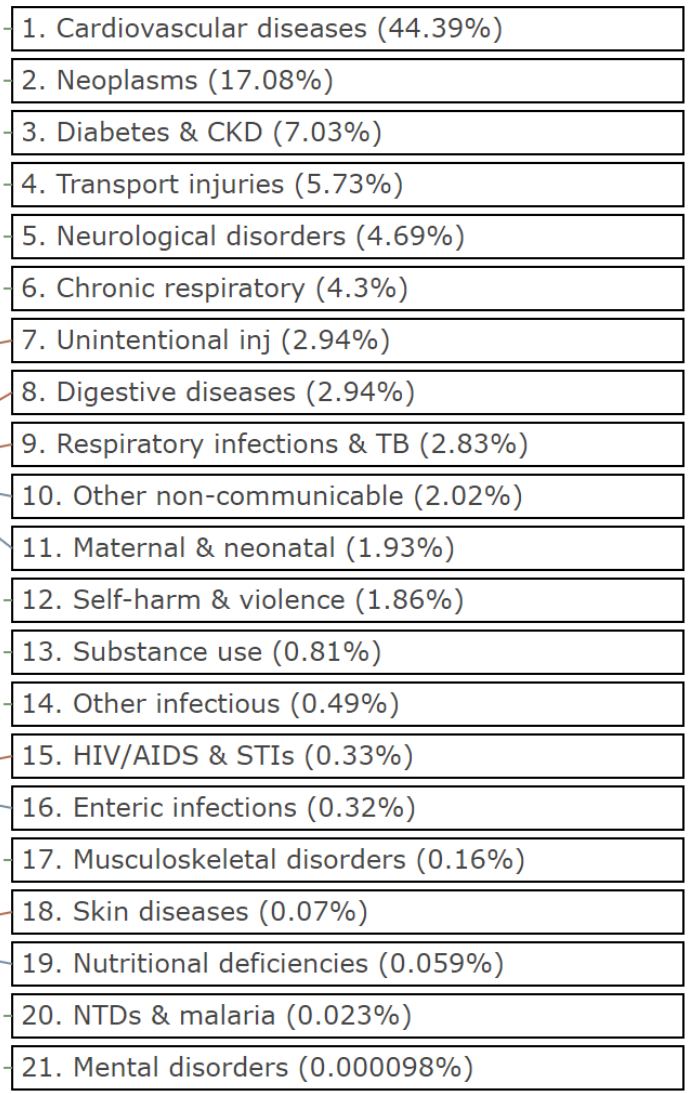

Figure 3. Causes of Death (Percent of Total Deaths) from the Global Burden of Disease (GBD) Study, 2015 vs. 2019. 
Table 2. Share of Different Causes of Death in Iran Based on NOCR Released Data and GBD Estimates (2019)

\begin{tabular}{|c|c|c|c|}
\hline GBD & & NOCR & \\
\hline $\begin{array}{l}\text { Respiratory infections \& } \\
\text { tuberculosis }\end{array}$ & \multirow{5}{*}{$3.99 \%$} & \multirow{5}{*}{ A00-B99 } & \multirow{5}{*}{$4.96 \%$} \\
\hline Other infectious & & & \\
\hline HIV/AIDS \& STIS & & & \\
\hline Enteric infections & & & \\
\hline NTDs \& malaria & & & \\
\hline Neoplasms & $17.08 \%$ & C00-D48 & $11.54 \%$ \\
\hline Hemoglobinopathies* & $0.01 \%$ & D50-D89 & $37.85 \%$ \\
\hline Diabetes* & \multirow{2}{*}{$3.83 \%$} & \multirow{2}{*}{ E00-E90 } & \multirow{2}{*}{$2.27 \%$} \\
\hline Nutritional deficiencies & & & \\
\hline Substance use & \multirow{2}{*}{$0.81 \%$} & \multirow{2}{*}{ F00-F99 } & \multirow{2}{*}{$1.14 \%$} \\
\hline Mental disorders & & & \\
\hline Neurological disorders & $4.69 \%$ & G00-G99 & $0.92 \%$ \\
\hline Cardiovascular disease & $44.39 \%$ & $100-199$ & $7.45 \%$ \\
\hline Chronic respiratory & $4.30 \%$ & J00-J99 & $0.66 \%$ \\
\hline Digestive diseases & $2.94 \%$ & К00-К93 & $2.18 \%$ \\
\hline Skin diseases & $0.07 \%$ & L00-L99 & $0.32 \%$ \\
\hline Musculoskeletal disorders & $0.16 \%$ & M00-M99 & $1.23 \%$ \\
\hline Chronic kidney disease* & \multirow{4}{*}{$3.41 \%$} & \multirow{4}{*}{ N00-N99 } & \multirow{4}{*}{$10.53 \%$} \\
\hline Urinary diseases* & & & \\
\hline Acute glomerulonephritis* & & & \\
\hline Gynecological diseases* & & & \\
\hline Maternal disorders* & $0.06 \%$ & O00-O99 & $2.30 \%$ \\
\hline Neonatal disorders* & $1.88 \%$ & P00-P96 & $0.15 \%$ \\
\hline Congenital defects* & $1.33 \%$ & Q00-Q99 & $0.02 \%$ \\
\hline Sudden infant death syndrome & $0.03 \%$ & R00-R99 & $0.03 \%$ \\
\hline Transport injuries & \multirow{3}{*}{$10.53 \%$} & \multirow{3}{*}{ V01-X59 } & \multirow{3}{*}{$10.19 \%$} \\
\hline Unintentional injury & & & \\
\hline Self-harm \& violence & & & \\
\hline Other & $0.41 \%$ & Other & $6.27 \%$ \\
\hline Total & $100 \%$ & Total & $100 \%$ \\
\hline \multicolumn{4}{|c|}{$\begin{array}{l}\text { GBD, Global Burden of Disease; NOCR, National Organization for } \\
\text { Civil Registration; HIV, human immunodeficiency virus; AIDS, acquired } \\
\text { immunodeficiency syndrome; STIs, sexually transmitted infections; NTDs } \\
\text { neglected tropical diseases. } \\
\text { * "level 3" cause of death in the GBD study (causes without an * in the table } \\
\text { above are "level 2" in the GBD study). }\end{array}$} \\
\hline
\end{tabular}

the cause of death is one of the common errors on death certificates. ${ }^{17,20}$ Phrases such as cardiac arrest that describe the immediate cause of death can be due to a wide range of underlying causes; therefore, they are not valid causes of death and should be considered as garbage codes. ${ }^{18}$ The significant reduction in codes I00-I99 from 2018 may have resulted from implementing a new algorithm to identify and exclude some of the common garbage codes. However, assigning only $7.45 \%$ of total deaths in Iran to ICD codes I00-199 is an underrepresentation of cardiovascular mortality. Other studies have estimated cardiovascular diseases as the cause of more than $40 \%$ of deaths in Iran. ${ }^{21,22}$ The reduction in R00-R99 codes is in line with the national targets under the CRVS Decade goals, but it seems implausible to occur in one year (2017 to 2018). Such significant changes in causes of deaths need to be flagged and require further assessment of the methodology used by NOCR. We did not find enough methodological information alongside the report on causes of death to explain potential reasons for these dramatic changes.
Unlike the NOCR cause of death data, the GBD estimations, which are based on data from MOHME and a modeling approach, do not show any major change in causes of death in Iran between 2015 and 2019. This is compatible with our hypothesis that the significant changes after 2017 in the NOCR report are due to a technical issue.

Completeness and timeliness of NOCR mortality data are its biggest strengths; through the COVID-19 pandemic, the NOCR mortality data showed an increase in the number of deaths (excess deaths) that was much higher than the official MOHME reports on COVID-19 deaths. ${ }^{23,24}$ However, the completeness needs to be validated by direct or indirect methods at both national and provincial levels.

In conclusion, timely release of causes of death data from registered deaths is promising for public health and medical professionals; however, the reports need to be clear and robust on methods. A linkage between NOCR death registration data and MOHME cause of death system could improve both systems. The two organizations have a similar experience for linkage of NOCR birth registry and the Iranian Maternal and Neonatal Network (IMAN) system. On the other hand, causes of death data need to contain a minimum level of details, at least 3-digit ICD codes; ICD chapters are noticeably big categories and need to be split into smaller groups.

\section{Authors' Contribution}

$M M L, H T$, and $M N$ conceived the idea for the study. HT and MML analyzed the data and drafted the manuscript. MN critically appraised the draft. All authors approved the final manuscript for publication.

\section{Conflict of Interest Disclosures}

The authors have no conflict of interest to declare.

\section{Ethical Statement}

This article is based on publicly available deidentifed data. The Institutional Review Board of the Iran University of Medical Sciences exempted the study from review.

\section{Supplementary Materials}

Supplementary file 1 contains Tables e1-e31.

\section{References}

1. World Health Organization (WHO). Civil registration and vital statistics (CRVS). Available from: https://www.who.int/ healthinfo/civil_registration/en/. Accessed February 26, 2021.

2. Centers for Disease Control and Prevention (CDC). National Vital Statistics System Improvements. 2020 April. Available from: https://www.cdc.gov/nchs/about/factsheets/factsheet_ nvss_improvements.htm. Accessed February 26, 2021.

3. ACOG Committee Opinion No. 748 Summary: The Importance of Vital Records and Statistics for the ObstetricianGynecologist. Obstet Gynecol. 2018;132(2):537-8. doi: 10.1097/aog.0000000000002760.

4. Centers for Disease Control and Prevention (CDC). Global Program for Civil Registration and Vital Statistics (CRVS) Improvement. 2015 November. Available from: https://www. cdc.gov/nchs/isp/isp_crvs.htm. Accessed February 26, 2021.

5. United Nations Economic and Social Commission for Asia 
and the Pacific. Asian and Pacific Civil Registration and Vital Statistics Decade 2015-2024 Booklet. Bangkok: United Nations Publications; 2015. Available from: https://www.unescap.org/ sites/default/files/Asian_and_Pacific_Civil_Registration_and_ Vital_Statistics_Decade2015-2024_Booklet.pdf.

6. National Organization for Civil Registration (NOCR) Office of Statistics, Demographic Information \& Immigration. 1395 SH Population Statistics Yearbook. Tehran: National Organization for Civil Registration; 2017. Persian. Available from: https://www. sabteahval.ir/DeskTopModules/Magazines/showattachment. aspx?id=3307\&mld=49823.

7. International statistical classification of diseases and related health problems. 10th rev., 5th ed. Vol. 2. France: World Health Organization; 2016. ISBN: 9789241549165 . Available from: https://icd.who.int/browse10/Content/statichtml/ ICD10Volume2_en_2019.pdf.

8. National Organization for Civil Registration (NOCR). The history of "The National Organization for Civil Registration". n.d. Available from: https://www.sabteahval.ir/en/History. Accessed February 26, 2021.

9. National Organization for Civil Registration (NOCR) Office of Statistics, Demographic Information \& Immigration. 1385 SH Population Statistics Yearbook. Tehran: National Organization for Civil Registration; 2007. Persian. Available from: https://www. sabteahval.ir/DeskTopModules/Magazines/showattachment. aspx?id=3294\&mld=49823.

10. Alipour A, Khodakarim S, Khosravi A, Ataey A. Estimating population cause-specific mortality fractions in the Islamic Republic of Iran: validation of Murray's method. East Mediterr Health J. 2020;26(3):290-6. doi: 10.26719/emhj.19.031.

11. Kazemi E, Khosravi A, Aghamohamadi S, Shariati M, Kazemeini $\mathrm{H}$. Trends in premature mortality in the Islamic Republic of Iran: probability of dying between ages 30 and 70 years. East Mediterr Health J. 2021;27(1):33-40. doi: 10.26719/emhj.20.067.

12. National Organization for Civil Registration (NOCR) Office of Statistics, Demographic Information \& Immigration. 1397 SH Population Statistics Yearbook. Tehran: National Organization for Civil Registration; 2019. Persian. Available from: https://www. sabteahval.ir/DeskTopModules/Magazines/showattachment. aspx?id=3308\&mld=49823.

13. National Organization for Civil Registration (NOCR). Vital Statistics Yearly Summary 2015-19. 2020. Available from: https://www.sabteahval.ir/DeskTopModules/Magazines/ showattachment.aspx?id=3370\&mld=49823.
14. Global Burden of Disease. Lancet; n.d. Available from: https:// www.thelancet.com/gbd. Accessed February 26, 2021.

15. Vos T, Lim SS, Abbafati C, Abbas KM, Abbasi M, Abbasifard $\mathrm{M}$, et al. Global burden of 369 diseases and injuries in 204 countries and territories, 1990-2019: a systematic analysis for the Global Burden of Disease Study 2019. Lancet. 2020;396(10258):1204-22. doi: 10.1016/s01406736(20)30925-9.

16. Institute for Health Metrics and Evaluation (IHME). GBD Compare. Seattle, WA: IHME, University of Washington; 2019. Available from: https://vizhub.healthdata.org/gbdcompare/. Accessed February 26, 2021.

17. Brooks EG, Reed KD. Principles and pitfalls: a guide to death certification. Clin Med Res. 2015;13(2):74-82; quiz 3-4. doi: 10.3121/cmr.2015.1276.

18. National Organization for Civil Registration (NOCR) Office of Statistics, Demographic Information \& Immigration. $1396 \mathrm{SH}$ Population Statistics Yearbook. Tehran: National Organization for Civil Registration; 2018. Persian. Available from: https://www. sabteahval.ir/DeskTopModules/Magazines/showattachment. aspx?id=3309\&mld $=49823$.

19. Global Health Estimates 2016: Deaths by Cause, Age, Sex, by Country and by Region, 2000-2016. Geneva: World Health Organization; 2018. Available from: https://www.who.int/ healthinfo/global_burden_disease/estimates/en/. Accessed February 26, 2021.

20. PrittBS, Hardin NJ, Richmond JA, Shapiro SL. Death certification errors at an academic institution. Arch Pathol Lab Med. 2005;129(11):1476-9. doi: 10.5858/2005-129-1476-dceaaa.

21. Moradi-Lakeh M, Sepanlou SG, Karimi SM, Khalili N, Djalalinia S, Karimkhani C, et al. Trend of socio-demographic index and mortality estimates in Iran and its neighbors, 19902015; findings of the Global Burden of Diseases 2015 Study. Arch Iran Med. 2017;20(7):419-28.

22. World Health Organization (WHO). Noncommunicable Diseases (NCD) Country Profiles 2018. Geneva: World Health Organization; 2018. ISBN: 9789241514620 . Available from: https://apps.who.int/iris/handle/10665/274512.

23. Tadbiri H, Moradi-Lakeh M, Naghavi M. All-cause excess mortality and COVID-19-related deaths in Iran. Med I Islam Repub Iran. 2020;34:80. doi: 10.34171/mjiri.34.80.

24. Tadbiri H, Moradi-Lakeh $M$, Naghavi $M$. Letter to the editor: COVID-19 and all-cause excess mortality in Iran in spring 2020. Med J Islam Repub Iran. 2020;34:125. doi: 10.34171/ mjiri.34.125. 